\title{
Hankel forms and sums of random variables
}

\author{
by \\ Henry Helson (Berkeley)
}

\begin{abstract}
A well known theorem of Nehari asserts on the circle group that bilinear forms in $H^{2}$ can be lifted to linear functionals on $H^{1}$. We show that this result can be extended to Hankel forms in infinitely many variables of a certain type. As a corollary we find a new proof that all the $L^{p}$ norms on the class of Steinhaus series are equivalent.
\end{abstract}

1. Hankel forms. A Hankel form in $\ell^{2}$ is one of the form

$$
\langle a, b\rangle=\sum_{j, k=0}^{\infty} a_{j} b_{k} \varrho_{j+k}
$$

where $\left(\varrho_{n}\right)(n \geq 0)$ is a square-summable sequence. It can be written

$$
\sum_{k=0}^{\infty} \sum_{j=0}^{k} a_{j} b_{k-j} \varrho_{k} .
$$

From this it is easy to see that if

$$
f\left(e^{i x}\right)=\sum a_{k} e^{i k x}, \quad g\left(e^{i x}\right)=\sum b_{k} e^{i k x}
$$

then the value of the sum (1.1) depends on the function $f g$, but not on $f$ and $g$ individually. Furthermore if $\left(f_{n}\right),\left(g_{n}\right)$ is a finite collection of functions in $H^{2}(T)$ ( $T$ is the circle group) with coefficients respectively $\left(a_{n k}\right),\left(b_{n k}\right)$ such that

$$
\sum f_{n} g_{n}
$$

is 0 , then

$$
\sum_{n} \sum_{k=0}^{\infty} \sum_{j=0}^{k} a_{n j} b_{n, k-j} \varrho_{k}=0 .
$$

2000 Mathematics Subject Classification: 43A15, 30B50, 15 A63.

Key words and phrases: Hankel form, Hilbert-Schmidt form, homogeneous Fourier series. 
Thus the form defines a linear functional on the subspace of $H^{1}(T)$ spanned by such products $f g$. Actually every function of $H^{1}(T)$ is a product of two functions in $H^{2}(T)$, so the functional is defined on $H^{1}(T)$ itself. It follows that there is a function $\phi$ in $L^{\infty}(T)$ such that $\widehat{\phi}(k)=\varrho_{k}(k \geq 0)$. This is a well known result of Nehari. The converse is easy: every bounded function $\phi$ leads to a bounded Hankel form by the reverse route.

The recent evolution of the theory of Dirichlet series leads to questions about analogous statements for forms in many variables. Let $K$ be the infinite-dimensional torus whose dual $\Gamma$ is realized as the subgroup of the line (in discrete topology) consisting of all real numbers $\log r$, where $r$ is a positive rational number. If $r$ has the prime factoring

$$
r=\prod p_{j}^{n_{j}}
$$

where $p_{1}=2, p_{2}=3, \ldots$ are the prime integers, then the character $\log r$ has values

$$
\chi_{\log r}\left(e^{i x_{1}}, e^{i x_{2}}, \ldots\right)=\exp \left(i \sum n_{j} x_{j}\right) .
$$

Thus $\Gamma$ can also be viewed as the group of sequences $\left(n_{1}, n_{2}, \ldots\right)$ of integers terminating in zeros.

The narrow cone $\Lambda$ in $\Gamma$ consists of all $\log r$ such that each $n_{j}$ is nonnegative; that is, $r$ is a positive integer. $H^{p}(K)(p \geq 1)$ consists of all functions $f$ in $L^{p}(K)$ whose Fourier series are sums over the narrow cone:

$$
f\left(e^{i x_{1}}, \ldots\right) \sim \sum_{\text {all } n_{j} \geq 0} a\left(n_{1}, \ldots\right) e^{i \sum n_{j} x_{j}} .
$$

This is a power series in the variables $z_{j}=e^{i x_{j}}(j=1,2, \ldots)$. It depends on infinitely many variables, but each term only contains a finite number of them.

Addition in $\Gamma$ is addition of corresponding components of sequences $\left(n_{1}, \ldots\right)$. This mirrors multiplication of the corresponding rational numbers $r$ given by (1.6). Thus it is natural to define a Hankel form in infinitely many variables to be a form

$$
\langle a, b\rangle=\sum_{j, k \geq 1} a_{j} b_{k} \varrho_{j k}
$$

where $a, b$ are square-summable sequences indexed by the positive integers, the kernel $\varrho$ is a square-summable sequence, and $j k$ is a product of integers, not a double subscript. If $\phi$ is a bounded function on $K$ and $\widehat{\phi}(\log n)=\varrho_{n}$ $(n \geq 1)$ then the form is bounded. We ask whether the converse, the analogue of Nehari's theorem, is true. It is no longer the case that every function of $H^{1}(K)$ is the product of functions in $H^{2}(K)$, so the argument given above does not apply. Nevertheless the form defines a linear functional at least in the part of $H^{1}(K)$ spanned by products $g h$, where $g, h$ belong to $H^{2}(K)$, 
exactly as recounted for the circle group above, and if a bounded function $\phi$ realizes this functional, then $\phi$ is lifted from the form. If every bounded Hankel form of some class can be lifted we shall say the class has the lifting property.

In [5] the lifting property was connected to another question. Denote by $\mathcal{K}$ the linear set of finite sums (1.4), where the factors all belong to $H^{2}(K)$. In $\mathcal{K}$ we define the tensor norm

$$
\|h\|=\inf \sum\left\|f_{n}\right\|_{2}\left\|g_{n}\right\|_{2}
$$

where the infimum extends over all finite sums (1.4) equal to $h$. Thus $\mathcal{K}$ is a subspace of $H^{1}(K)$ with a larger norm. Perhaps the completion of $\mathcal{K}$, which we denote by $\mathcal{K}^{*}$, is all of $H^{1}(K)$. It is if and only if the tensor norm on $\mathcal{K}$ is equivalent to the norm in $H^{1}(K)$. We do not know whether this is the case, but

The class of all bounded Hankel forms has the lifting property if and only if $\mathcal{K}^{*}=H^{1}(K)$.

The equivalence, stated in [5], is easy to establish.

2. Hilbert-Schmidt forms. A Hankel form with kernel $\varrho$ is of HilbertSchmidt type if

$$
\sum_{j, k=1}^{\infty}\left|\varrho_{j k}\right|^{2}<\infty
$$

Then the form is bounded, with bound at most the square root of the sum. The terms of the sum are the same for all pairs $(j, k)$ such that the product $j k$ has a given value $n$. Therefore the sum in (2.1) is the same as

$$
\sum_{n=1}^{\infty}\left|\varrho_{n}\right|^{2} d(n)
$$

where $d(n)$ is the number of divisors of $n$. (For example, if $n$ is prime then $d(n)=2$.)

Our main result is this.

THEOREM. The class of Hilbert-Schmidt Hankel forms in infinitely many variables has the lifting property.

This answers Question 3 of [5, p. 54]. It does not settle the question raised above, but has its own consequences.

The proof rests on the following result about the circle.

Lemma 1. For $f$ in $H^{1}(T)$ with Fourier coefficients $a_{n}$,

$$
\left(\sum_{n=0}^{\infty}\left|a_{n}\right|^{2} /(n+1)\right)^{1 / 2} \leq\|f\|_{1} .
$$


The convergence of the sum on the left is of course a weaker statement than the convergence of the sum in the better known inequality

$$
\sum_{n=0}^{\infty}\left|a_{n}\right| /(n+1) \leq \pi\|f\|_{1}
$$

of Hardy and Littlewood [4, p. 129] (the bound $\pi$ was found by I. Schur); but for us the bound in (2.3) is essential, and it is due to Vukotić [8].

Throughout this paper, the Lebesgue spaces are constructed with normalized Lebesgue measure, which we denote generically by $\sigma$ on various torus groups.

Here is a proof of (2.3). We may assume that $f$ has norm 1 . Factor $f$ as $g h$ with $g, h$ in $H^{2}(T)$ and $|g|=|h|$, so that $\|g\|_{2}=\|h\|_{2}=1$. Let $g$ and $h$ have Fourier coefficients $\left(b_{n}\right)$ and $\left(c_{n}\right)$, respectively. Then the left side of $(2.3)$ is the supremum over sequences $\left(e_{n}\right)$ of

$$
\sum_{n=0}^{\infty}\left|a_{n}\right| e_{n} / \sqrt{n+1}=\sum_{n=0}^{\infty}\left|\sum_{j=0}^{n} b_{j} c_{n-j}\right| e_{n} / \sqrt{n+1}
$$

where the $e_{n}$ are non-negative, square-summable, with squared-sum equal to 1 . If we replace the $b_{n}$ and $c_{n}$ by their moduli the expression on the right is increased, so we may take them to be non-negative. Setting $n=j+k$ transforms (2.5) to

$$
\sum_{j, k=0}^{\infty} b_{j} c_{k} e_{j+k} / \sqrt{j+k+1}
$$

This is a Hankel form of Hilbert-Schmidt type, whose bound is at most the square root of

$$
\sum_{j, k=0}^{\infty} e_{j+k}^{2} /(j+k+1) .
$$

For each non-negative integer $m$ there are $m+1$ terms with $j+k=m$; therefore $(2.7)$ is

$$
\sum_{m=0}^{\infty} e_{m}^{2}=1
$$

This shows that the left side of (2.5) is at most 1 , and the lemma is proved.

F. Bayart has proved this result $[1,2,5]$ : for $f$ in $H^{1}(K)$, and $n=\prod p_{j}^{n_{j}}$,

$$
\sum\left|\widehat{f}\left(n_{1}, \ldots\right)\right|^{2} / n^{\varepsilon}<\infty
$$


for every positive $\varepsilon$. We are going to prove that

$$
\left(\sum_{n=1}^{\infty}\left|\widehat{f}\left(n_{1}, \ldots\right)\right|^{2} / d(n)\right)^{1 / 2} \leq\|f\|_{1}
$$

The statement of our theorem will follow easily by duality. This improves Bayart's theorem in two respects: $d(n)=O\left(n^{\varepsilon}\right)$ for every positive $\varepsilon$, and indeed $d(n)$ is much smaller than $n^{\varepsilon}$ if $n$ is prime or has few factors; and secondly, the precise bound given by (2.10) has no analogue in (2.9). But our proof of (2.10) will be identical with the proof of Bayart, substituting Lemma 1 above for a different piece of information.

The proof will be given in the next section; first we state a needed inequality, introduced into this subject by A. Bonami [3]:

LEMMA 2. Let $\varrho$ be a positive function on the product of spaces $X$ and $Y$ carrying measures $d x, d y$. Then

$$
\left(\int\left(\int \varrho(x, y) d x\right)^{2} d y\right)^{1 / 2} \leq \int\left(\int \varrho^{2}(x, y) d y\right)^{1 / 2} d x
$$

This is an integral version of Minkowski's inequality. Note that the order of integration is reversed by the inequality! (A proof is given in [5].)

3. Proof of the theorem. Let $n$ have the prime factoring (1.6). All the divisors of $n$ are obtained by replacing each $n_{j}$ by all the $k_{j}$ satisfying $0 \leq k_{j} \leq n_{j}$. Hence the number of divisors of $n$ is exactly

$$
\prod_{j}\left(n_{j}+1\right) \text {. }
$$

Of course only finitely many $n_{j}$ are different from 0 .

We repeat Bayart's argument. The statement to be proved is

$$
\left(\sum_{\text {all } n_{j} \geq 0} \frac{\left|\widehat{f}\left(n_{1}, n_{2}, \ldots\right)\right|^{2}}{\left(n_{1}+1\right)\left(n_{2}+1\right) \ldots}\right)^{1 / 2} \leq\|f\|_{1}
$$

It will suffice to prove this for all $f$ that are analytic trigonometric polynomials. Lemma 1 is (3.2) for $f$ depending on only one variable. Suppose that $f$ depends on $k$ variables. For $m=1, \ldots, k$ let $T_{m}$ be the operator defined by

$$
T_{m} \sum a\left(n_{1}, \ldots\right) e^{i \sum n_{j} x_{j}}=\sum \frac{a\left(n_{1}, \ldots\right)}{\sqrt{n_{m}+1}} e^{i \sum n_{j} x_{j}} .
$$

Then (3.2) becomes

$$
\left\|T_{1} \cdots T_{k} f\right\|_{2} \leq\|f\|_{1} .
$$


Lemma 1, applied to the first variable, gives us

$$
\begin{aligned}
& \left(\int\left|T_{1} \cdots T_{k} f\right|^{2} d \sigma\left(x_{1}, \ldots, x_{k}\right)\right)^{1 / 2} \\
& \quad \leq\left(\int\left(\int\left|T_{2} \cdots T_{k} f\right| d \sigma\left(x_{1}\right)\right)^{2} d \sigma\left(x_{2}, \ldots x_{k}\right)\right)^{1 / 2}
\end{aligned}
$$

The next step uses Lemma 2; the right side is less than

$$
\int\left(\int\left|T_{2} \cdots T_{k} f\right|^{2} d \sigma\left(x_{2}, \cdots, x_{k}\right)\right)^{1 / 2} d \sigma\left(x_{1}\right)
$$

Now we have one fewer $T$ 's, and one variable removed from the inside integral. We apply Lemma 1 to the second variable, and so forth. After $k$ steps we have left $\|f\|_{1}$, and (2.10) is proved.

The dual of the operation that carries an analytic trigonometric polynomial $f$ depending on $k$ variables with the norm of $H^{1}(K)$ to $T_{1} \cdots T_{k} f$ in $H^{2}(K)$, which we have shown reduces norm, maps $H^{2}(K)$ into the dual of $H^{1}(K)$. This means that if $\varrho$ satisfies

$$
\sum_{n=1}^{\infty}\left|\varrho_{n}\right|^{2} d(n)<\infty
$$

then there is a bounded function $\phi$ on $K$ whose Fourier coefficients $\widehat{\phi}\left(n_{1}, \ldots\right)$ are $\varrho_{n}$. This is the statement of the theorem.

4. Homogeneous Fourier series. The theorem has an unexpected application to some results that are usually treated in probability theory. A Steinhaus series $[6$, p. 134$]$ is a sum

$$
\sum_{n=1}^{\infty} a_{n} e^{i x_{n}}
$$

where the $x_{n}$ are independent real variables. These are the complex analogue of Rademacher series [6, p. 125], and we expect the same results about them. It is well known that all the $p$-norms are equivalent on such sums. This is a statement about Fourier series, and deserves a simple treatment in those terms. We shall show now that (2.10) contains this and other results.

TheOREM. Let $m$ be a positive integer, and

$$
f(x) \sim \sum_{\sum n_{j}=m} a\left(n_{1}, \ldots\right) e^{i \sum n_{j} x_{j}}
$$

the Fourier series of a function $f$ belonging to $H^{1}(K)$ and homogeneous of degree $m$. Then $f$ belongs to $H^{q}(K)$ for every finite $q$. For $q=2$ we have

$$
\|f\|_{2} \leq 2^{m / 2}\|f\|_{1} \text {. }
$$

If $m=2$ then $\exp |f|$ is summable. 
The first statement was proved by Bayart [2]. The bound in the second statement is not the best possible; in a difficult paper, Sawa [7] shows for $m=1$ (the case of Steinhaus series) that the best bound is $2 / \pi^{1 / 2}$.

According to $(2.10)$, for $f$ in $H^{1}(K)$

$$
\left(\sum\left|\widehat{f}\left(n_{1}, \ldots\right)\right|^{2} / d(n)\right)^{1 / 2} \leq\|f\|_{1}
$$

If $n$ is the product of $m$ distinct primes, then $d(n)=2^{m}$. If some of the primes are repeated $d(n)$ is smaller. Therefore for $f$ with Fourier series (4.2)

$$
\left(\sum\left|\widehat{f}\left(n_{1}, \ldots\right)\right|^{2} / 2^{m}\right)^{1 / 2} \leq\|f\|_{1}
$$

which is (4.3).

Now $f^{2}$ is summable, and its Fourier series is also homogeneous (of degree $2 m)$, so $f^{2}$ is also square-summable, and so on. Therefore $f$ belongs to $H^{q}(K)$ for every finite $q$.

When we keep track of the bounds at each step, we find that

$$
\|f\|_{n} \leq n^{m / 2}\|f\|_{1}
$$

when $n$ is a power of 2 . For other values of $n$ the next power of 2 is less than $2 n$, so that

$$
\|f\|_{n} \leq(2 n)^{m / 2}\|f\|_{1}
$$

for all positive integers $n$. The expansion

$$
e^{\lambda|f|}=\sum_{n=0}^{\infty} \lambda^{n}|f|^{n} / n !
$$

converges in the norm of $L^{1}(K)$ for positive $\lambda$ such that

$$
\sum_{n=0}^{\infty} \lambda^{n}\|f\|_{n}^{n} / n !<\infty .
$$

Hadamard's formula relates the radius of convergence of this series to

$$
\lim \sup \left[\log \|f\|_{n}-\frac{1}{n} \sum_{k=1}^{n} \log k\right] .
$$

By comparing the sum with an integral we have

$$
\frac{1}{n} \sum_{k=1}^{n} \log k>\log n-1 .
$$

From this fact and (4.7), (4.10) is less than

$$
\limsup \left[\log \|f\|_{1}+\frac{m}{2}(\log n+\log 2)-\log n+1\right] .
$$


For $m=2$ the bracket is the constant $\log \|f\|_{1}+\log 2+1$. Thus the radius of convergence is large if $\|f\|_{1}$ is small.

The argument finishes in the conventional way. By omitting the first terms in (4.2) the norm of $f$ in $L^{2}(K)$ can be made as small as we please. All the norms are equivalent, so the norm of $f$ in $L^{1}(K)$ is small too, and (4.12) can be made negatively large, and (4.9) converges for large $\lambda$. The omitted terms are a bounded function, and the proof is finished.

The same proof (as pointed out by H. Queffélec) leads to the statement that for any positive integer $m, \exp |f|^{2 / m}$ is summable if $f$ is homogeneous of degree $m$.

Acknowledgements. I would like to express my appreciation to J. E. McCarthy and to H. Queffélec for correspondence about the subject of this paper.

\section{References}

[1] F. Bayart, Hardy spaces of Dirichlet series and their composition operators, Monatsh. Math. 136 (2002), 203-236.

[2] - Opérateurs de composition sur des espaces de séries de Dirichlet et problèmes d'hypercyclicité simultanée, thèse, Université des Sciences et Technologies de Lille, 2002.

[3] A. Bonami, Étude des coefficients de Fourier des fonctions de $L^{p}(G)$, Ann. Inst. Fourier (Grenoble) 20 (1970), 335-402.

[4] H. Helson, Harmonic Analysis, 2nd ed., published by the author, 1995.

[5] - Dirichlet Series, published by the author, 2005.

[6] S. Kaczmarz and H. Steinhaus, Theorie der Orthogonalreihen, Chelsea reprint, 1951.

[7] J. Sawa, Best constant in the Khintchine inequality for complex Steinhaus variables, the case $p=1$, Studia Math. 81 (1985), 107-126.

[8] D. Vukotić, The isoperimetric inequality and a theorem of Hardy and Littlewood, Amer. Math. Monthly 110 (2003), 532-536.

Mathematics Department

University of California

Berkeley, CA 94720-3840, U.S.A.

E-mail: hhelson@aol.com 